\title{
Predictive Factors for Response to Neoadjuvant Therapy in Breast Cancer
}

\author{
Sibylle Loibl ${ }^{a, b}$ Gunter von Minckwitz ${ }^{a, c}$ Michael Untch ${ }^{d}$ Carsten Denkert ${ }^{\mathrm{e}}$ on behalf of the \\ German Breast Group
}

${ }^{a}$ German Breast Group, Neu-Isenburg, Germany

${ }^{\text {b }}$ Sana-Klinikum, Offenbach, Germany

' University Women's Hospital, Frankfurt, Germany

${ }^{d}$ HELIOS-Kliniken Berlin-Buch, Frauenklinik, Interdisziplinäres Brustzentrum, Berlin, Germany

${ }^{\mathrm{e}}$ Institut für Pathologie der Charité, Berlin, Germany

\section{Keywords \\ Breast cancer - Neoadjuvant therapy · Biomarker . Predictor}

\section{Summary}

Within 2 decades, neoadjuvant therapy (NAT) has become a standard treatment option in breast cancer. The advantage of NAT is the ability to monitor the treatment effect. Pathological complete response (pCR) after NAT is a very good predictor for long-term outcome. Clinical factors, such as age and body mass index, as well as recently identified biomarkers can predict the chance of achieving a pCR. Hormone-receptor status, proliferation markers, immune infiltrates and genetic alterations, such as germline BRCA and PIK3CA, can now be measured almost on a routine basis due to the decreased analysis costs.

\section{Introduction}

Neoadjuvant therapy (NAT) is 1 option of standard therapy in breast cancer (BC) $[1,2]$. Although the proportion of patients achieving a pathological complete response (pCR) to neoadjuvant (chemo)therapy has increased, $40-80 \%$ of the patients, depending on the biological subtype of their tumour, do not achieve a pCR. It has been consistently demonstrated that $\mathrm{pCR}$ is very good prognostic factor for long-term benefit from NAT, especially for triple-negative (TNBC) and HER2positive $\mathrm{BC}$ [3-6]. As in the adjuvant setting, the absolute benefit from chemotherapy is smaller for hormone receptor-positive $(\mathrm{HR}+)$ tumours, resulting in a lower pCR rate. Nevertheless, HR+ patients who, irrespective of their HER2 status, achieve a pCR have a better disease-free (DFS) and overall survival (OS) compared to patients who do not attain pCR. Despite pCR holding strong prognostic information, there are patients with pCR who relapse (small number) and patients who do not achieve pCR and never experience a relapse (large number). Therefore, other tools with a more refined separation of the patients into groups with a lower and a higher risk of relapse are needed. The CPS-EG score has been constructed and validated as a prognostic tool, taking into consideration the tumour burden before and after therapy as well as the oestrogen receptor and the grading [7, 8]. Selecting patients with resistance to a given therapy prior to the start of therapy would be the ultimate goal.

Generally, identification of patients with a higher or a lower chance of achieving a pCR can be based on clinical factors and, in the era of translational research, biomarkers.

\section{Clinical Factors}

Among the clinical factors, age is an important predictor of response. Young patients seem to achieve higher $\mathrm{pCR}$ rates after NAT [9] (fig. 1 [10]). In the GeparTrio study, age was the only independent predictive factor for achieving a pCR in TNBC. The most important factor for survival in TNBC is pCR. In HR+/HER2+ tumours, age also seems to provide prognostic information in patients achieving a pCR [10]. Body mass index (BMI) and age are closely related [11]. Patients with a high BMI have a significantly lower chance of achieving a $\mathrm{pCR}$, and patients with a normal BMI also have the best DFS and OS.

Patients who have an early clinical response to chemotherapy after the first 2-4 cycles have a significantly higher pCR

\section{KARGER \\ Fax +497614520714 \\ Information@Karger.com}

www.karger.com

\section{(c) 2014 S. Karger GmbH, Freiburg}

2296-5270/14/3710-0563\$39.50/0

Accessible online at:

www.karger.com/ort
Prof. Dr. med. Sibylle Loibl

German Breast Group

c/o GBG Forschungs GmbH

Martin-Behaim-Str. 12, 63263 Neu-Isenburg, Germany

sibylle.loibl@germanbreastgroup.de 
Fig 1. Age as predictor to neoadjuvant therapy.

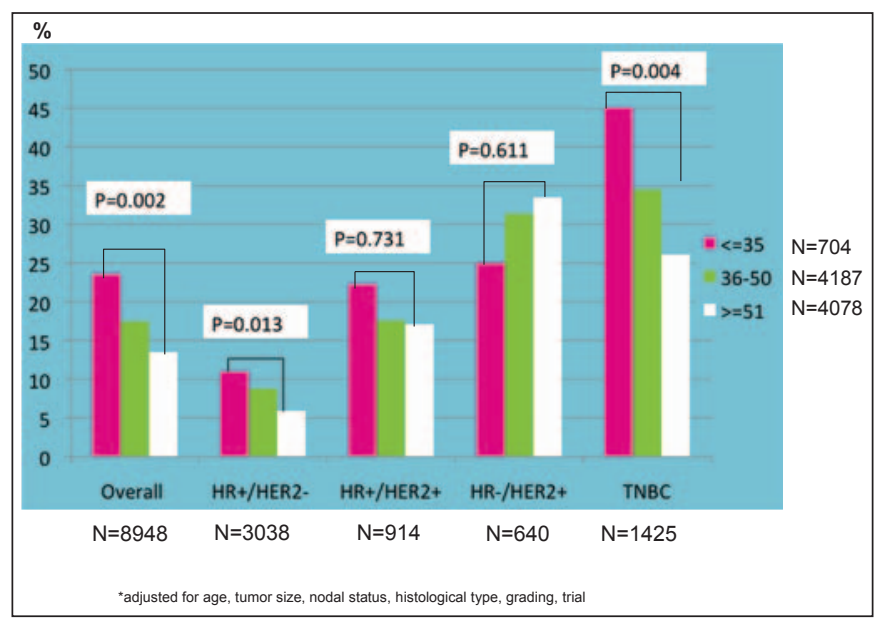

rate than those not responding to the first 2-4 cycles. Overall, the responding groups achieve a pCR of about $22 \%$, whereas the non-responding patients have a pCR rate of only about $5 \%[12,13]$. Several trials have studied whether the adaption of treatment according to early response has an impact on response and/or survival [14-16]. The GeparTrio study [9, 12,13] investigated whether intensifying therapy at the time of early response is superior to standard therapy. Intensifying the therapy in the responding patients as well as changing the treatment in the non-responding patients resulted in an improved DFS and OS, even though the pCR was not increased. However, this approach seems only to apply for the patients with an $\mathrm{HR}+$ tumour. For patients with a HR-negative (HR-) tumour, the most important prognostic factor is pCR, and adapting the treatment according to response did not have an effect.

\section{Biomarkers}

Recently identifying biomarkers for predicting response and resistance has become an important research objective in NAT. The therapeutic landscape has become increasingly complex, and thus the necessity to select the right treatment for the patients is crucial [17]. The HR status is still 1 of the most important biomarkers in BC. In almost all clinical trials investigating neoadjuvant treatment, the HR status was predictive for lower response but better survival [3]. To find additional markers for TNBC, we and others have investigated the androgen receptor (AR). This marker is an independent prognostic marker for DFS and OS in general [18]. Patients with an AR+ tumour had a significantly lower pCR rate than those with an AR- tumour [19]. In TNBC there was no difference in regard to $\mathrm{pCR}$ between androgen-receptor positive or negative patients in this study, but others found a difference in favour of higher $\mathrm{pCR}$ rate for TNBC patients who were also $\mathrm{AR}$ - compared to AR+ TNBC patients [20].
Fig 2. Tumour-associated lymphocytes.

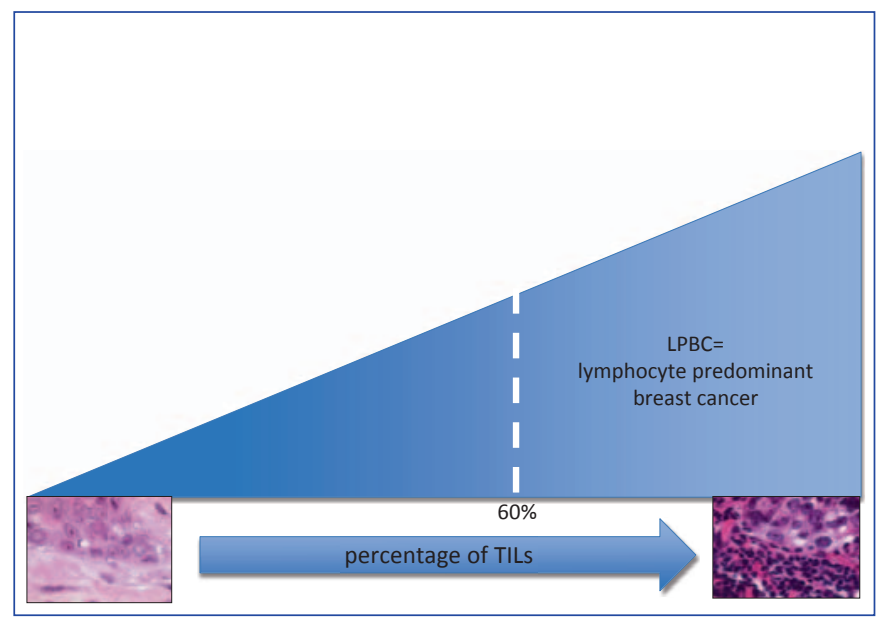

Proliferation is probably the most important factor predicting a pCR after NAT, and different surrogates for proliferation have been put forward. Tumour grade has been shown to be an independent predictor for a pCR at surgery with grade 3 tumours being most favourable [9]. The highest pCR rates were detected in young patients $(<40$ years) with undifferentiated tumours. Subgroup analysis revealed that grading remained predictive in luminal A and B tumours. Surrogate intrinsic subtyping using tumour grading has even been shown to outperform intrinsic subtyping in terms of response prediction [21]. The proliferation marker Ki67 is a valuable tool that provides predictive information regarding NAT response and prognosis across molecular subgroups [22]. Being a continuous variable, numerous cut-off points have been defined for Ki67 to try and discern those with a better from those with a worse prognosis [23, 24]. An issue that remains under debate is the different performance of the marker regarding its predictive and prognostic value in different molecular subgroups. In HR+ tumours, the Ki67 cut-off point of $14 \%$ has been validated as distinguishing prognostically good (luminal A) and prognostically poor (luminal B) subtypes [25], and has been used for recommendations [26]. However, the cut-off point is rather arbitrary, and a cut-off off of $20 \%$ has been put forward instead [27].

The genomic grade index (GGI) is a gene expression signature that has been shown to outperform the classic histological grade assessment in terms of prediction, and classifies tumour specimen into low or high risk instead of grades 1,2 , or 3. In the HER2-normal setting, high GGI was significantly and independently predictive for increased chemotherapy response regardless of the HR status [28]. However, despite higher response rates, patients with high GGI had a worse prognosis in terms of distant DFS if they were also oestrogen positive, a relationship that had already been seen when using histological grading as a predictor.

The recurrence score (RS) is a panel of 21 genes that was set up to quantify recurrence risk in oestrogen-positive, node- 
negative patients [29]. The RS has also been described to correlate with $\mathrm{pCR}$, such that patients with high RS, and therefore high recurrence risk, are also those most likely to benefit from chemotherapy [30]. Similarly, the ROR (risk of relapse score), which is based on the PAM 50 (the prediction analysis of microarray 50) assay, has been investigated within the NOAH study and was found to be highly predictive of response to neoadjuvant chemotherapy. As shown for the RS, patients with high ROR showed higher pCR rates than those with low or intermediate ROR [31]. Another approach to assess probability of response to treatment is the use of immune markers, due to the suggested effect of the immune system to enhance chemotherapy effectiveness [32].

Tumour-infiltrating lymphocytes (TILs) have been proposed to provide information on $\mathrm{pCR}$ prediction in a number of studies. TILs can be grouped into intratumoural (i.e. those with direct contact to tumour cells), stromal (i.e. those between the tumour cells) and LPBC (lymphocyte predominant $\mathrm{BC}$, i.e. if there are more lymphocytes than tumour cells). Lymphocyte infiltration used as a continuous factor (fig. 2) is predictive of neoadjuvant chemotherapy response, and tumour specimens classified as LPBC had significantly increased pCR rates compared to non-LPBC $[33,34]$. Moreover, an independent association between TILs and higher responses to trastuzumab and chemotherapy was confirmed in primary HER2+ disease, and an underlying correlation between TILs and immune genes has been found [35]. TILs have also shown to be indicative of good prognosis after chemotherapy, particularly in TNBC [36]. The role of LPBC was also investigated within the translational research programme of the recent GeparSixto study, in which previous results on the predictive value of LBPC in TNBC and HER2+ were validated [37]. There was a significant difference in pCR rates between no LPBC and LPBC groups in the overall cohort. This was even more pronounced in patients treated with carboplatin, indicating a real predictive effect for carboplatin in HER2 + BC. This needs to be validated. In addition, it was shown that the up-regulation of immune genes (immune-suppressing as well as immune-stimulating genes) predict pCR [38].

\section{Molecular Subgroup-Specific Predictors}

TNBC is heterogeneous and lacks the possibility of targeted therapies. However, TNBC is also special in that, with the use of more sophisticated and combined therapies, pCR rates have continuously increased in recent years [9, 39-42]. TNBC has been subcategorized into 6 specific and 1 unknown group, and all of them lead to different responses to neoadjuvant chemotherapy [20,43], the basal-like 1 subgroup showing the highest $\mathrm{pCR}$ rate with $52 \%$ and basal-like 2 and luminal AR group the lowest rate with only $0 \%$ and $10 \%$, respectively. This classification warrants prospective validation but has already set hopes high for distinct response prediction in TNBC.
$B R C A$ mutations have been linked with higher risk for TNBC and seem to be a good predictor of response to NAT for $\mathrm{BC}$. It has been shown that carriers of the BRCA1 mutation had significantly higher $\mathrm{pCR}$ rates compared to $B R C A 2$ carriers or non-carriers, irrespective of the NAT administered [44]. The GeparSixto study recently demonstrated not only a higher $\mathrm{pCR}$ rate for mutation carriers and those with a positive family history, but also a stronger benefit from carboplatin, still to be confirmed in larger cohorts [45].

In HER2+ disease pCR rates have increased dramatically over the years due to the advancement in treatment possibilities. The use of trastuzumab, longer chemotherapy regimens and lately the dual anti-HER2 therapy have helped to continuously raise the pCR rate to as much as $50-60 \%$. The recent Neo-ALTTO study showed a significantly higher pCR rate in the dual anti-HER2 treatment group (51.3\%) compared with trastuzumab $(29.5 \%)$ or lapatinib $(24.7 \%)$ alone [46] and, furthermore, that pCR correlated with better survival [47].

In the light of these results the importance of detecting the target correctly becomes obvious. Prediction of pCR can only be reliable if patients are treated adequately according to their true HER2 status. Analysis of the GeparQuattro data revealed relevant differences between local and central HER2 testing and the consequences in terms of $\mathrm{pCR}$ prediction. Centrally HER2+ patients showed the largest benefit from anti-HER2 therapy, with a pCR rate of $46.8 \%$, whereas patients that were locally HER2+ but negative in the central evaluation had only a $20.3 \%$ probability of obtaining a pCR [48].

Within the HER2+ study population, HR status has been identified as a predictor for pCR after NAT. Subgroup analysis of the NeoALTTO and the NeoSphere trial data showed that the benefit in terms of pCR rate was higher in HR- patients compared with $\mathrm{HR}+$ patients, independent of the antiHER2 therapy (lapatinib, trastuzumab or combination) given $[47,49]$. The same effect was observed in the NSABP B-41 trial in which even higher $\mathrm{pCR}$ rates could be achieved using the combination of weekly paclitaxel and targeted therapy (lapatinib, trastuzumab or combination) following standard doxorubicin plus cyclophosphamide treatment [50].

With p95HER2 (p95), a promising biomarker has been detected that might predict response to NAT for BC in HER2+ patients. P95 is the C-terminal fragment of the full-length HER2 and can be detected by an immunohistochemical assay using a monoclonal antibody that specifically recognizes the 611 C-terminal fragment. A translational effort using samples from the GeparQuattro study has shown that tumours staining positive for p95 (20\% cut-off) showed a higher pCR rate following chemotherapy plus trastuzumab than p95- tumours (59\% vs $24 \%, \mathrm{p}=0.001)$. The predictive value of $\mathrm{p} 95$ appeared more pronounced in $\mathrm{HR}+$ tumours and was independent from age, tumour and nodal stage, grade and HR status [51]. These intriguing results were, however, not supported by the data from the smaller CHER-LOB study, in which p95 was not found to be predictive for pCR in any treatment group [52]. 
More recently, the PIK3CA gene has been in the focus as another promising marker [53]. A landmark study by Samuels et al. [54] found frequent tumour-specific (somatic) mutations in PIK3CA, the gene encoding $\mathrm{p} 110 \alpha 62$, in various human cancers, with $>90 \%$ of the mutations affecting exon 9 and 20 [55]. PIK3CA has been found to be the second most frequently mutated gene in $\mathrm{BC}$, with unequal distribution amongst the different biological subtypes [56]. Mutations in HER2-overexpressing cells have been shown to predict resistance to trastuzumab but not to lapatinib therapy [57]. In the same study, a loss of the phosphatase and tensin homolog (PTEN) predicted for response to lapatinib. The predictive value of PIK3CA was confirmed using much larger sample sizes within the GeparQuattro, GeparQuinto and GeparSixto trials. $P I K 3 C A$ was mutated in $20.8 \%$ of HER2+ tumours [58]. The pCR rate was significantly lower in the PIK3CA mutant compared to the wild-type cohort in HER2+ tumours, with the difference being largest when a double HER2 blockade was present [58]. The lowest pCR rate of only $6.3 \%$ was found within an HER2+/HR + cohort harbouring a PIK3CA mutation, identifying HR status in addition to PIK3CA mutation as an independent predictor of pCR in HER2+ BC. Those results are in concordance with data from the Neo-ALTTO [59], Neosphere [60] and the TBCRC 006 [61] studies, and the concept has paved the way for the development of new treatment options with $P I 3 K$-targeting agents. In the phase II Neophoebe study (NCT01816594), HER2-+ patients are stratified according to their PIK3CA mutation status and randomized within each cohort to neoadjuvant trastuzumab versus trastuzumab + BKM120 (a potent and highly specific oral pan-class I PI3K inhibitor) in combination with weekly paclitaxel. Results for the primary endpoint pCR will reveal if the PIK3CA mutant population benefits more from inhibition of the PI3K pathway.

Another putative predictive marker, TP53, which is associated with DNA damage response, has been evaluated in the NAT setting. In a study using a taxane-based regimen, TP53 did not offer reliable prediction of a pCR after 2 chemotherapy cycles [62]. Likewise, an evaluation of whether taxanes confer a greater advantage than anthracyclines in tumours with mutated TP53 compared with wild-type TP53 found that, despite being prognostic for OS, TP53 was not predictive of preferential sensitivity to taxanes [63].

In summary, several factors have been identified that can be used to predict response following systemic NAT and this is reflected in the current $\mathrm{AGO}$ guidelines (www.ago-online. de). Promising new candidates, such as TILs, immune markers, PIK3CA mutation or the Vanderbilt signature, as well as the germline $B R C A$ status are still under investigation. However, there is still a lack of predictors when bevacizumab, dosedense regimens or PARP inhibitors are being used, and further research will be needed in these areas.

\section{Disclosure Statement}

The authors have no conflict of interests. The contrent was partly presented as keynote lecture at the conference of the German Cancer Society (DKG) from 19.-22. February, 2014 in Berlin. Parts of the work presented by the group were funded within the EU 7th framework programme; grant no. 278659 'RESPONSIFY'.

\section{References}

$>1$ Mauri D, Pavlidis N, Ioannidis JP: Neoadjuvant versus adjuvant systemic treatment in breast cancer: A meta-analysis. J Natl Cancer Inst 2005;97: 188-194.

2 Kaufmann M, Hortobagyi GN, Goldhirsch A, et al.: Recommendations from an international expert panel on the use of neoadjuvant (primary) systemic treatment of operable breast cancer: An update. J Clin Oncol 2006;24:1940-1949.

3 von Minckwitz G, Untch M, Blohmer JU, et al.: Definition and impact of pathologic complete response on prognosis after neoadjuvant chemotherapy in various intrinsic breast cancer subtypes. $\mathrm{J}$ Clin Oncol 2012;30:1796-1804.

4 Liedtke C, Mazouni C, Hess KR, et al.: Response to neoadjuvant therapy and long-term survival in patients with triple-negative breast cancer. J Clin Oncol 2008;26:1275-1281.

$\checkmark 5$ Untch M, Fasching PA, Konecny GE, et al.: Pathologic complete response after neoadjuvant chemotherapy plus trastuzumab predicts favorable survival in human epidermal growth factor receptor 2-overexpressing breast cancer: results from the TECHNO trial of the AGO and GBG study groups. J Clin Oncol 2011;29:3351-3357.

-6 Cortazar P, Zhang L, Untch M, et al.: Pathological complete response and long-term clinical benefit in breast cancer: the CTNeoBC pooled analysis. Lancet 2014;384:164-172.
7 Jeruss JS, Mittendorf EA, Tucker SL, et al.: Combined use of clinical and pathologic staging variables to define outcomes for breast cancer patients treated with neoadjuvant therapy. J Clin Oncol 2008;26:246-252.

$>8$ Mittendorf EA, Jeruss JS, Tucker SL, et al.: Validation of a novel staging system for disease-specific survival in patients with breast cancer treated with neoadjuvant chemotherapy. J Clin Oncol 2011;29: 1956-1962.

9 Huober J, von Minckwitz G, Denkert C, et al.: Effect of neoadjuvant anthracycline-taxane-based chemotherapy in different biological breast cancer phenotypes: overall results from the GeparTrio study. Breast Cancer Res Treat 2010;124:133-140.

10 Loibl S, Jackisch C, Gade S, et al.: Neoadjuvant chemotherapy in the very young 35 years of age or younger. Cancer Res 2012;72(suppl 24):S3-1.

11 Fontanella C, von Minckwitz G, Mergler B, et al.: Body mass index (BMI) and treatment outcome of breast cancer patients receiving neoadjuvant therapy (NACT). Cancer Res 2013;73:PD2-2:

12 von Minckwitz G, Kümmel S, Vogel P, et al.: Neoadjuvant vinorelbine-capecitabine versus docetaxel-doxorubicin-cyclophosphamide in early nonresponsive breast cancer: Phase III randomized GeparTrio trial. J Natl Cancer Inst 2008; 100:542-551.
13 von Minckwitz G, Kümmel S, Vogel P, et al.: Intensified neoadjuvant chemotherapy in early-responding breast cancer: Phase III randomized GeparTrio study. J Natl Cancer Inst 2008;100:552-562.

14 von Minckwitz G, Blohmer JU, Costa SD, et al. Response-guided neoadjuvant chemotherapy for breast cancer. J Clin Oncol 2013;31:3623-3630.

15 Smith IC, Heys SD, Hutcheon AW, et al.: Neoadjuvant chemotherapy in breast cancer: Significantly enhanced response with docetaxel. J Clin Oncol 2002;20:1456-1466.

16 Heys SD, Hutcheon AW, Sarkar TK, et al.: Neoadjuvant docetaxel in breast cancer: 3-year survival results from the Aberdeen trial. Clin Breast Cancer 2002;3 (suppl 2):S69-74.

17 Higgins MJ, Baselga J: Targeted therapies for breast cancer. J Clin Invest 2011;121:3797-3803.

18 Vera-Badillo FE, Templeton AJ, de Gouveia P, et al.: Androgen receptor expression and outcomes in early breast cancer: A systematic review and metaanalysis. J Natl Cancer Inst 2014;106:djt319.

19 Loibl S, Müller BM, von Minckwitz G, et al.: Androgen receptor expression in primary breast cancer and its predictive and prognostic value in patients treated with neoadjuvant chemotherapy. Breast Cancer Res Treat 2011;130:477-487. 
20 Masuda H, Baggerly KA, Wang Y, et al.: Differential response to neoadjuvant chemotherapy among 7 triple-negative breast cancer molecular subtypes. Clin Cancer Res 2013;19:5533-5540.

-21 Lips EH, Mulder L, de Ronde JJ, et al.: Breast cancer subtyping by immunohistochemistry and histological grade outperforms breast cancer intrinsic subtypes in predicting neoadjuvant chemotherapy response. Breast Cancer Res Treat 2013;140:63-71.

-22 Fasching PA, Heusinger K, Haeberle L, et al.: Ki67, chemotherapy response, and prognosis in breast cancer patients receiving neoadjuvant treatment. BMC Cancer 2011;11:486.

23 Denkert C. Blohmer JU, Müller BM, et al.: Ki67 levels in pretherapeutic core biopsies as predictive and prognostic parameters in the neoadjuvant GeparTrio trial. Cancer Res 2012;72(suppl 24):102s.

24 Denkert C, Loibl S, Müller BM, et al.: Ki67 levels as predictive and prognostic parameters in pretherapeutic breast cancer core biopsies: A translational investigation in the neoadjuvant GeparTrio trial. Ann Oncol 2013;24:2786-2793.

25 Cheang MC, Chia SK, Voduc D, et al.: Ki67 index, HER2 status, and prognosis of patients with luminal B breast cancer. J Natl Cancer Inst 2009;101:736-750.

26 Goldhirsch A, Wood WC, Coates AS, et al.: Strategies for subtypes - dealing with the diversity of breast cancer: Highlights of the St. Gallen International Expert Consensus on the Primary Therapy of Early Breast Cancer 2011. Ann Oncol 2011;22: 1736-1747.

27 Goldhirsch A, Winer EP, Coates AS, et al.: Personalizing the treatment of women with early breast cancer: Highlights of the St. Gallen International Expert Consensus on the Primary Therapy of Early Breast Cancer 2013. Ann Oncol 2013;24:2206-2223.

28 Liedtke C, Hatzis C, Symmans WF, et al.: Genomic grade index is associated with response to chemotherapy in patients with breast cancer. J Clin Oncol 2009;27:3185-3191.

29 Paik S, Shak S, Tang G, et al.: A multigene assay to predict recurrence of tamoxifen-treated, nodenegative breast cancer. N Engl J Med 2004;351: 2817-2826.

-30 Gianni L, Zambetti M, Clark K, et al.: Gene expression profiles in paraffin-embedded core biopsy tissue predict response to chemotherapy in women with locally advanced breast cancer. J Clin Oncol 2005;23:7265-7277.

-31 Prat A, Bianchini G, Thomas M, et al.: Researchbased PAM50 subtype predictor identifies higher responses and improved survival outcomes in HER2-positive breast cancer in the NOAH study. Clin Cancer Res 2014;20:511-521.

-32 Zitvogel L, Kepp O, Kroemer G: Immune parameters affecting the efficacy of chemotherapeutic regimens. Nat Rev Clin Oncol 2011;8:151-160.

33 Denkert C, Loibl S, Noske A, et al.: Tumor-associated lymphocytes as an independent predictor of response to neoadjuvant chemotherapy in breast cancer. J Clin Oncol 2010;28:105-113.

-34 Issa-Nummer Y, Darb-Esfahani S, Loibl S, et al. Prospective validation of immunological infiltrate for prediction of response to neoadjuvant chemotherapy in HER2-negative breast cancer - a substudy of the neoadjuvant GeparQuinto trial. PLoS One 2013;8:e79775.

-35 Loi S, Michiels S, Salgado R, et al.: Tumor infiltrating lymphocytes (TILs) indicate trastuzumab benefit in early-stage HER2-positive breast cancer (HER2+ BC). Cancer Res 2013;73:S1-05.

-36 Loi S, Sirtaine N, Piette F, et al.: Prognostic and predictive value of tumor-infiltrating lymphocytes in a phase III randomized adjuvant breast cancer trial in node-positive breast cancer comparing the addition of docetaxel to doxorubicin with doxorubicin-based chemotherapy: BIG 02-98. J Clin Oncol 2013;31:860-867.

37 Denkert C, Loibl S, Salat C, et al.: Increased tumor-associated lymphocytes predict benefit from addition of carboplatin to neoadjuvant therapy for triple-negative and HER2-positive early breast cancer in the GeparSixto trial (GBG 66). Cancer Res 2013;73:S1-06.

38 Denkert C, von Minckwitz G, Brase JC, et al.: Expression of immunologic genes in triple-negative and HER2-positive breast cancer in the neoadjuvant GEPARSIXTO trial: Prediction of response to carboplatin-based chemotherapy. J Clin Oncol 2014;32:5s(suppl):abstr 510

39 von Minckwitz G, Eidtmann H, Rezai M, et al.: Neoadjuvant chemotherapy and bevacizumab for HER2-negative breast cancer. N Engl J Med 2012;366:299-309.

40 Gerber B, Loibl S, Eidtmann H, et al.: Neoadjuvant bevacizumab and anthracycline-taxanebased chemotherapy in 678 triple-negative primary breast cancers; results from the geparquinto study (GBG 44). Ann Oncol 2013;24:2978-2984.

41 von Minckwitz G, Schneeweiss A, Loibl S, et al.: Neoadjuvant carboplatin in patients with triplenegative and HER2-positive early breast cancer (GeparSixto; GBG 66): A randomised phase 2 trial. Lancet Oncol 2014;15:747-756.

42 Sikov WM, Berry DA, Perou CM, et al.: Impact of the addition of carboplatin and/or bevacizumab to neoadjuvant once-per-week paclitaxel followed by dose-dense doxorubicin and cyclophosphamide on pathologic complete response rates in stage II to III triple-negative breast cancer: CALGB 40603 (alliance). J Clin Oncol 2014;pii: JCO.2014.57.0572.

43 Lehmann BD, Bauer JA, Chen X, et al.: Identification of human triple-negative breast cancer subtypes and preclinical models for selection of targeted therapies. J Clin Invest 2011;121:27502767.

-44 Arun B, Bayraktar S, Liu DD, et al.: Response to neoadjuvant systemic therapy for breast cancer in BRCA mutation carriers and noncarriers: A single-institution experience. J Clin Oncol 2011;29: 3739-3746.

45 von Minckwitz G, Hahnen E, Fasching PA, et al.: Pathological complete response (pCR) rates after carboplatin-containing neoadjuvant chemotherapy in patients with germline BRCA (gBRCA) mutation and triple-negative breast cancer (TNBC): Results from GeparSixto. J Clin Oncol 2014;32:5s(suppl): abstr 1005 .

46 Baselga J, Bradbury I, Eidtmann H, et al.: Lapatinib with trastuzumab for HER2-positive early breast cancer (NeoALTTO): A randomised, open-label, multicentre, phase 3 trial. Lancet 2012;379:633-640.

47 de Azambuja E, Holmes AP, Piccart-Gebhart M, et al.: Lapatinib with trastuzumab for HER2-positive early breast cancer (NeoALTTO): survival outcomes of a randomised, open-label, multicentre, phase 3 trial and their association with pathological complete response. Lancet Oncol 2014;pii: S1470-2045(14)70320-1.

48 Denkert C, Huober J, Loibl S, et al.: HER2 and ESR1 mRNA expression levels and response to neoadjuvant trastuzumab plus chemotherapy in patients with primary breast cancer. Breast Cancer Res 2013;15:R11.

49 Gianni L, Pienkowski T, Im YH, et al.: Efficacy and safety of neoadjuvant pertuzumab and trastu- zumab in women with locally advanced, inflammatory, or early HER2-positive breast cancer (NeoSphere): A randomised multicentre, openlabel, phase 2 trial. Lancet Oncol 2012;13:25-32.

50 Robidoux A, Tang G, Rastogi P, et al.: Lapatinib as a component of neoadjuvant therapy for HER2-positive operable breast cancer (NSABP protocol B-41): An open-label, randomised phase 3 trial. Lancet Oncol 2013;14:1183-1192.

51 Loibl S, Bruey J, von Minckwitz G, et al.: Validation of p95 as a predictive marker for trastuzumab-based therapy in primary HER2-positive breast cancer: A translational investigation from the neoadjuvant GeparQuattro study. J Clin Oncol 2011;29:(suppl): abstr 530.

52 Guarneri V, Frassoldati A, Bottini A, et al. Preoperative chemotherapy plus trastuzumab, lapatinib, or both in human epidermal growth factor receptor 2-positive operable breast cancer: results of the randomized phase II CHER-LOB study. J Clin Oncol 2012;30:1989-1995.

53 Berenjeno IM, Vanhaesebroeck B: PI3K regulatory subunits lose control in cancer. Cancer Cell 2009;16:449-450.

54 Samuels Y, Wang Z, Bardelli A, et al.: High frequency of mutations of the PIK3CA gene in human cancers. Science 2004;304:554

55 Loi S, Michiels S, Lambrechts D, et al.: Somatic mutation profiling and associations with prognosis and trastuzumab benefit in early breast cancer. J Natl Cancer Inst 2013;105:960-967.

56 Cancer Genome Atlas Network: Comprehensive molecular portraits of human breast tumours. Nature 2012;490:61-70.

57 Dave B, Migliaccio I, Gutierrez MC, et al.: Loss of phosphatase and tensin homolog or phosphoinositol-3 kinase activation and response to trastuzumab or lapatinib in human epidermal growth factor receptor 2-overexpressing locally advanced breast cancers. J Clin Oncol 2011;29:166-173.

58 Loibl S, von Minckwitz G, Schneeweiss A, et al.: PIK3CA mutations are associated with lower rates of pathological complete response (pCR) to anti-HER2 therapy in primary HER2-overexpressing breast cancer. J Clin Oncol 2014, in press.

59 Baselga J, Majewski I, Nuciforo PG, et al.: PI3KCA mutations and correlation with pCR in the NeoALTTO trial (BIG 01-06). Eur J Cancer 2013;49:S402.

60 Gianni L, Bianchini G, Kiermaier A, et al.: Neoadjuvant pertuzumab $(\mathrm{P})$ and trastuzumab $(\mathrm{H})$ : Biomarker analyses of a 4-arm randomized phase II study (NeoSphere) in patients (pts) with HER2positive breast cancer (BC). Cancer Res 2011; 71(suppl 24):S5-1.

61 Contreras A, Herrera S, Wang T, et al.: PIK3CA mutations and/or low PTEN predict resistance to combined anti-HER2 therapy with lapatinib and trastuzumab and without chemotherapy in TBCRC006, a neoadjuvant trial of HER2-positive breast cancer patients. Cancer Res 2013;73 (suppl 24):PD1-2.

62 von Minckwitz G, Sinn HP, Raab G, et al.: Clinical response after two cycles compared to HER2, Ki$67, \mathrm{p} 53$, and bcl-2 in independently predicting a pathological complete response after preoperative chemotherapy in patients with operable carcinoma of the breast. Breast Cancer Res 2008;10: R30.

63 Bonnefoi H, Piccart M, Bogaerts J, et al.: TP53 status for prediction of sensitivity to taxane versus non-taxane neoadjuvant chemotherapy in breast cancer (EORTC 10994/BIG 1-00): a randomised phase 3 trial. Lancet Oncol 2011;12:527-539. 